\title{
Dynamic Behavior Analysis and Robust Synchronization of a Novel Fractional-Order Chaotic System with Multiwing Attractors
}

\author{
Chenhui Wang (iD) \\ College of Applied Mathematics, Xiamen University of Technology, Xiamen 361024, China \\ Correspondence should be addressed to Chenhui Wang; chwang@xmut.edu.cn
}

Received 27 November 2020; Revised 29 December 2020; Accepted 31 March 2021; Published 12 April 2021

Academic Editor: Heng Liu

Copyright (c) 2021 Chenhui Wang. This is an open access article distributed under the Creative Commons Attribution License, which permits unrestricted use, distribution, and reproduction in any medium, provided the original work is properly cited.

To enrich the types of multiwing chaotic attractors in fractional-order chaotic systems (FOCSs), a new type of 3-dimensional FOCSs is designed in this study. The most important contribution of this FOCS consists in the coexistence of multiple multiwing chaotic attractors, including 2-wing, 3-wing, and 4-wing attractors. It is also indicated that the minimum order that the system can exhibit chaotic behavior is 0.84 . Then, based on certain fractional stability criteria, a robust synchronization controller is derived for this kind of FOCSs with multiwing chaotic attractors and parametric uncertainties, and the stability of the synchronization error is proven strictly. Meanwhile, the theoretical analysis is tested by simulation results.

\section{Introduction}

Recently, the promotion of fractional-order systems (FOSs) based on integer-order dynamic systems (IODSs) has provided better mathematical models for many physical and engineering systems. FOSs have many dynamic behaviors similar to IODSs, such as chaos, bifurcation, and attractors. Meanwhile, the chaos control and synchronization have shown potential applications in physics, biology, information, dynamics control, and other fields. In the 1940s, Scott and Gerasimov established a fractional differential solid model between Newtonian fluids and Hooke's law [1]. Capoto applied fractional calculus to the study of complex viscoelastic mechanics and rheological media and gave a fractional differential mechanical model [1]. Baglay and Torvik established a viscoelastic material model based on fractional differential [2]. In 1983, B. B. Mandport pointed out the fact that there are a large number of fractal dimensions in nature and many domains in science and technology, and there is a self-similar phenomenon between the dynamic systems described by integer-order calculus and fractional calculus theory [3]. In particular, the discovery of the self-similarity of the whole and part, the close connection between the fractional Brownian motion and the Riemann-Liouville FC, and the theory and application of fractional operators have developed rapidly in the world. Since Pecora and Carroll discovered that two chaotic systems can be synchronized in 1990, the chaos of chaotic synchronization has been ignited. Synchronous control of fractional-order chaotic systems (FOCSs) has become a research hotspot because it has potential application prospects in secure communication and other fields. So chaotic synchronization is very important, but there are certain difficulties. There are already some synchronization methods in this respect, such as active control $[4,5]$, nonlinear state observer method [6,7], sliding mode control method, and adaptive feedback control method [8-10]. However, the above methods are all applied to a class of FOCSs, and few general methods can be applied to any FOCSs.

Meanwhile, as a generalization of traditional controllers, fractional-order controllers (FOCs) have attracted more and more attention because of their flexibility and integrity. Tiltintegral-derivative (TID) controller, $\mathrm{PI}^{\lambda} \mathrm{D}^{\mu}$ controller, and CRNOE controller are all well-known FOCs $[5-7,11,12]$. They show more superior characteristics than traditional controllers in the control of chaotic systems. Han et al. used a sliding mode control strategy to achieve synchronization of uncertain FOCSs [13], where a new control method, i.e., composite learning method is used to achieve an accurate estimation of an uncertain term. Based on the idea of 
adaptive T-S fuzzy control, a new synchronization method is proposed for FOCSs in [14], where a new approximation theorem is proposed. Agrawal et al. used active control methods to achieve the synchronization of two different FOCSs in [15]. However, most of these chaotic synchronization methods are discussed based on certain FOCSs, without considering modeling errors, measurement errors, structural changes, environmental noise, and other factors. In practical applications, the uncertainty and external interference caused by these factors usually present in the system are inevitable, and these factors have an adverse effect on the quality and performance of synchronization.

It is worth noting that, compared with FOCSs with fixedwing chaotic attractors, FOCSs with multiple multiwing chaotic attractors exhibit more complex dynamic behavior and better performance. In secure communication and image encryption, such FOCSs have higher sequence complexity and larger key space and can improve system security performance. Therefore, it is of great value to find and construct low-dimensional FOCSs with multiple multiwing chaotic attractors. $\mathrm{Xu}$ et al. constructed a corresponding fractional-order memristive system based on a four-wing integer-order memristive chaotic system, and this system allows three-wing and three-wing, three-wing and four-wing chaotic attractors to coexist [16]. Borah and Roy designed a new FOCS with the coexistence of three-wing and four-wing chaotic attractors in [17]. Xian et al. constructed a two-wing and four-wing chaotic attractor coexisting FOCS in [18]. At present, there are still some challenges in constructing a FOCS with more types of multiwing chaotic attractors coexisting. In the existing literature, it is relatively rare to produce a three-dimensional FOCS in which twowing to four-wing chaotic attractors coexist.

Inspired by the above discussion, based on the works described in [16-18], in this paper, a new three-dimensional FOCS is designed, and its dynamic analysis is performed. When the fractional order $\alpha$ lies on [0.83, 0.98], the coexistence of chaotic attractors such as two wings, two wings, and four wings can be obtained. Just like the interesting work of Xu et al. [16], the designed system does not have nonsmooth nonlinear functions, and it is easier to implement with hardware circuits. The chaotic behavior of the system was further verified. Then, by using fractional-order Lyapunov stability theory, in the presence of unmatched parametric uncertainties, a robust synchronization controller is designed, and the stability is proven strictly.

\section{Preliminaries}

The fractional integral can be written as

$$
\mathscr{I}^{q} g(t)=\frac{1}{\Gamma(q)} \int_{0}^{t} \frac{g(\tau)}{(t-\tau)^{1-q}} \mathrm{~d} \tau,
$$

and the fractional derivative is written by

$$
\mathscr{D}^{\alpha} g(t)=\frac{1}{\Gamma(k-q)} \int_{0}^{t} \frac{g^{(k)}(\tau)}{(t-\tau)^{q+1-k}} \mathrm{~d} \tau,
$$

with $\alpha \in[k-1, k)(k \in \mathbb{N})$. In this paper, only $\alpha \in(0,1)$ is considered.

Lemma 1 (see [9]). Let $x(t) \in \mathbb{C}[0,+\infty)$, then one has

$$
\frac{1}{2} \mathscr{D}^{\alpha} x^{2}(t) \leq x(t) \mathscr{D}^{\alpha} x(t)
$$

Lemma 2 (see [10]). Suppose that $y \in \mathbb{C}^{1}\left[0, t_{0}\right]$ where $t_{0} \in \mathbb{R}^{+}$and $0<\alpha \leq 1$. The following results hold:

$$
\begin{aligned}
& \mathscr{D}^{-\alpha} \mathscr{D}_{t}^{\alpha} y=y-y(0), \\
& \mathscr{D}^{\alpha} \mathscr{D}^{-\alpha} y=y .
\end{aligned}
$$

Lemma 3 (see [15]). Let $c \in \mathbb{R}^{+}$, it holds $|z|-z \tan h(z / c) \leq 0.2785 c=c^{\prime}$.

In this paper, the following algorithm will be used to find a numerical solution of a fractional equation.

Let

$$
\left\{\begin{array}{l}
\mathscr{D}_{t}^{\alpha} z=g(t, z), \\
z(0)=z_{0} .
\end{array}\right.
$$

According to Lemma 2, (5) is rearranged by

$$
z=z_{0}+\frac{1}{\Gamma(\alpha)} \int_{0}^{t}(t-\tau)^{\alpha-1} g(\tau, z(\tau)) \mathrm{d} \tau .
$$

Denote $h=(T / N)$. Then, one can estimate (6) by $[15,16,18]$

$$
\begin{aligned}
z_{h}\left(t_{n+1}\right)= & z_{0}+\frac{h^{\alpha}}{\Gamma(\alpha+2)} g\left(t_{n}+1, z_{h}^{p}\left(t_{n+1}\right)\right) \\
& +\frac{h^{\alpha}}{\Gamma(\alpha+2)} \sum_{k=0}^{n} a_{k, n+1} g\left(t_{k}, z_{h}\left(t_{k}\right)\right),
\end{aligned}
$$

where $a_{k, n+1}=n^{\alpha+1}-(n-\alpha)(n+1)^{\alpha}$ for $k=0$ and $a_{k, n+1}=$ $(n-k+2)^{\alpha+1}+(n-k)^{\alpha+1}-2(n-k+1)^{\alpha+1}$ for $1 \leq k \leq n$, $z_{h}^{p}\left(t_{n+1}\right)=z_{0}+(1 / \Gamma(\alpha)) \sum_{k=0}^{n} b_{k, n+1} f\left(t_{k}, z_{h}\left(t_{k}\right)\right), p=\alpha+1$, and $b_{k, n+1}=\left(h^{\alpha} / \alpha\right)\left((n+1-k)^{\alpha}-(n-k)^{\alpha}\right)$.

Estimation error of this method is $\max \left|z\left(t_{k}\right)-z_{h}\left(t_{k}\right)\right|=$ $o\left(h^{p}\right)[19]$.

\section{Main Results}

3.1. Dynamics of a Novel FOCS. The Lü system is a famous chaotic system, which can be described by [20]

$$
\left\{\begin{array}{l}
\dot{x}=-\frac{b c}{b+c} x-y z+d, \\
\dot{y}=b y+x z \\
\dot{z}=c z+x y
\end{array}\right.
$$

with $b, c$, and $d$ are system parameters. To design a novel FOCS based on the above Lü system (8), let us add a nonlinear term $-q x-9 y z-d$ into the first equation of (8), add a term $-9 x y+y^{2}$ into the third equation of (8), and 
replace the conventional integer-order derivative by using a fractional derivative. Then, we can obtain the following FOCS [16]:

$$
\left\{\begin{array}{l}
\mathscr{D}^{\alpha} x=-a x-10 y z \\
\mathscr{D}^{\alpha} y=b y+x z \\
\mathscr{D}^{\alpha} z=c z-8 x y+y^{2}
\end{array}\right.
$$

with $a=-(b c /(b+c))-q, b+c \neq 0, b \neq 0, c \neq 0, a \neq 0$.

To discuss the dynamics behavior of the novel system (9), let us fix the system parameters first. Let $b=1.0, c=-0.78, q=1.5455$, then one has $a=2$. Thus, system (9) has five equilibriums:

$$
\begin{aligned}
& E_{1}:[0.3038,-0.1358,0.4472]^{T}, \\
& E_{2}:[0,0,0]^{T}, \\
& E_{3}:[-0.3038,0.1359,0.4472]^{T}, \\
& E_{4}:[-0.3214,-0.1437,-0.4472]^{T}, \\
& E_{5}:[0.3214,0.1437,-0.4472]^{T} .
\end{aligned}
$$

The Jacobian matrix is

$$
\mathbf{J}=\left[\begin{array}{ccc}
-2 & -10 z & -10 y \\
z & 1 & x \\
-8 y & 2 y-8 x & -0.78
\end{array}\right]
$$

and whose characteristic polynomial is given as

$$
F_{\lambda}=\lambda^{3}+a_{2} \lambda^{2}+a_{1} \lambda+a_{0},
$$

with $a_{2}=1.7812, a_{1}=8 x^{2}+10 z^{2}-80 y^{2}-2.0 x y-1.2221$, and $\quad a_{0}=16 x^{2}+80 y^{2}+7.8 z^{2}+20 y^{2} z-4 x y-160 x y z-$ 1.5612 .

Thus, the eigenvalues for five equilibriums are listed in Table 1.

To drive system (9) obtaining chaotic attractors, one should let $|\arg (\lambda)|<(\pi \alpha / 2)$. Thus, according to the values listed in Table 1, one knows that the minimal order is $q=0.84$.

When $\alpha=0.95$, the chaotic phenomenon of system (9) under different initial conditions is given in Figure 1, from which we can see that when the initial conditions are $[2,1,1]^{T}$ and $[5,1,1]^{T}$, there are 4-ring chaotic attractors (see Figures $1(\mathrm{a})-1(\mathrm{~d}))$. When the initial condition is $[11,1,1]^{T}$, only a 2-ring chaotic attractor exists (see Figures 1(e) and 1(f)).

When $\alpha=0.85$, the chaotic phenomenon of system (10) under different initial conditions is given in Figure 2, from which we can see that when the initial conditions are $[2,1,1]^{T}$ and $[11,1,1]^{T}$, there are 2 -ring chaotic attractors (see Figures 2(a)-2(c), 2(g)-2(i)). When the initial condition is $[11,1,1]^{T}$, a 4-ring chaotic attractor exists (see Figures 2(d)-2(f)).

It can be seen from the above theoretical analysis and simulation results that the chaotic characteristics of the system have a great relationship with the order and initial value of the system. In short, the system can exhibit very complex dynamic behavior.
TABLE 1: Eigenvalues of equilibriums.

\begin{tabular}{lccc}
\hline Equilibrium & $\lambda_{1}$ & $\lambda_{2}$ & $\lambda_{3}$ \\
\hline$E_{1}$ & -2.6358 & $0.4279-1.4997 i$ & $0.4279-1.4997 i$ \\
$E_{2}$ & -2 & -0.78 & 1 \\
$E_{3}$ & -2.6358 & $0.4279-1.4997 i$ & $0.4279-1.4997 i$ \\
$E_{4}$ & -2.6921 & $0.4562-1.4519 i$ & $0.4562+1.4519 i$ \\
$E_{5}$ & -2.6921 & $0.4562-1.4519 i$ & $0.4562+1.4519 i$ \\
\hline
\end{tabular}

3.2. Robust Synchronization of the FOCS. Let the FOCS (10) as the master FOCS. Taking parametric uncertainties into consideration, let the response of FOCS be

$$
\left\{\begin{array}{l}
\mathscr{D}^{\alpha} \widehat{x}=-a \widehat{x}-10 \widehat{y} \widehat{z}+u_{1}+\psi_{1}^{T} \theta_{1}, \\
\mathscr{D}^{\alpha} \widehat{y}=b \widehat{y}+\widehat{x} \widehat{z}+u_{2}+\psi_{2}^{T} \theta_{2}, \\
\mathscr{D}^{\alpha} \widehat{z}=c \widehat{z}-8 \widehat{x} \hat{y}+\widehat{y}^{2}+u_{3}+\psi_{3}^{T} \theta_{3},
\end{array}\right.
$$

where $\hat{x}, \hat{y}, \widehat{z} \in \mathbb{R}$ are the states of the slave FOCS, $u_{1}, u_{2}, u_{3} \in \mathbb{R}$ are the control inputs, $\psi_{1}(t), \psi_{2}(t)$, $\psi_{3}(t) \in \mathbb{R}^{m}$ are known vector functions, and $\theta_{1}(t), \theta_{2}(t)$, $\theta_{3}(t) \in \mathbb{R}^{m}$ are unknown constant vectors.

Let the synchronization error be $\varepsilon=\left[\varepsilon_{1}, \varepsilon_{2}, \varepsilon_{3}\right]^{T}=[x-\hat{x}, y-\widehat{y}, z-\widehat{z}]^{T} \in \mathbb{R}^{3}$. Thus, it follows from (9) and (13) that

$$
\left\{\begin{array}{l}
\mathscr{D}^{\alpha} \epsilon_{1}=-a \epsilon_{1}-10 y z+10 \widehat{y} \widehat{z}-u_{1}-\psi_{1}^{T} \theta_{1}, \\
\mathscr{D}^{\alpha} \epsilon_{2}=b \epsilon_{2}-\widehat{x} \hat{z}+x z-u_{2}-\psi_{2}^{T} \theta_{2}, \\
\mathscr{D}^{\alpha} \epsilon_{3}=c \epsilon_{3}-8 x y+8 \widehat{x} \hat{y}+y^{2}-\widehat{y}^{2}-u_{3}-\psi_{3}^{T} \theta_{3} .
\end{array}\right.
$$

Then, the controllers can be designed as

$$
\left\{\begin{array}{l}
u_{1}=\left(\sigma_{1}-a\right) \varepsilon_{1}+10 y z-10 \hat{y} \widehat{z}-\psi_{1}^{T} \widehat{\theta}_{1}-\tan h\left(\frac{\varepsilon_{1}}{\gamma_{1}}\right), \\
u_{2}=\left(b+\sigma_{2}\right) \varepsilon_{2}-\widehat{x} \widehat{z}+x z-\psi_{2}^{T} \widehat{\theta}_{2}-\tan h\left(\frac{\varepsilon_{2}}{\gamma_{2}}\right), \\
u_{3}=\left(c+\sigma_{3}\right) \varepsilon_{3}-8 x y+8 \hat{x} \hat{y}+y^{2}-\widehat{y}^{2}-\psi_{3}^{T} \widehat{\theta}_{3}-\tan h\left(\frac{\varepsilon_{3}}{\gamma_{3}}\right),
\end{array}\right.
$$

where $\sigma_{1}, \sigma_{2}, \sigma_{3}, \gamma_{1}, \gamma_{2}, \gamma_{3} \in \mathbb{R}^{+}$are constants.

Substituting (15) into (14) gives

$$
\mathscr{D}^{\alpha} \varepsilon_{i}=-\sigma_{i} \varepsilon_{i}-\tanh \left(\frac{\varepsilon_{i}}{\gamma_{i}}\right)+\psi_{i}^{T} \widetilde{\theta}_{i},
$$

where $i=1,2,3$ and $\widetilde{\theta}_{i}=\widehat{\theta}_{i}-\theta_{i}$ is an estimation error vector.

Then, it follows from (16) and Lemma 3 that

$$
\begin{aligned}
\varepsilon_{i} \mathscr{D}^{\alpha} \varepsilon_{i} & =-\sigma_{i} \varepsilon_{i}^{2}-\varepsilon_{i} \tanh \left(\frac{\varepsilon_{i}}{a_{i}}\right)-\varepsilon_{i} \psi_{i}^{T} \widetilde{\theta}_{i}, \\
& \leq-\sigma_{i} \varepsilon_{i}^{2}+\left|\varepsilon_{i}\right|-\varepsilon_{i} \tanh \left(\frac{\varepsilon_{i}}{a_{i}}\right)-\varepsilon_{i} \psi_{i}^{T} \widetilde{\theta}_{i} \\
& =-\sigma_{i} \varepsilon_{i}^{2}+0.2785 \gamma_{i}-\varepsilon_{i} \psi_{i}^{T} \widetilde{\theta}_{i} .
\end{aligned}
$$

The adaptation law is given by 


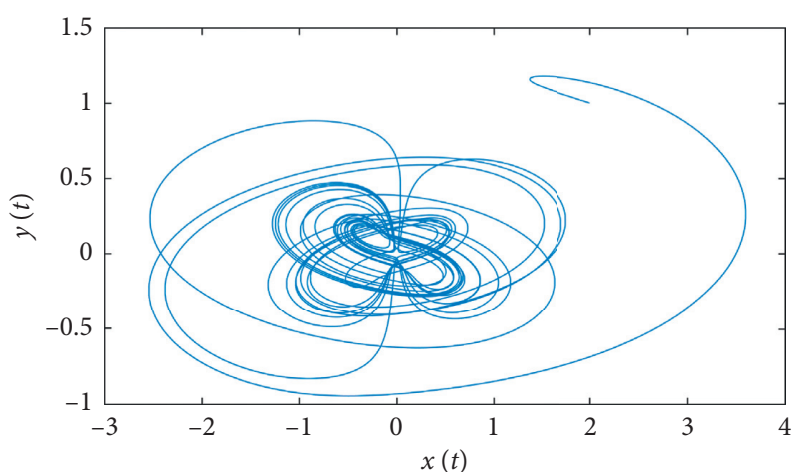

(a)

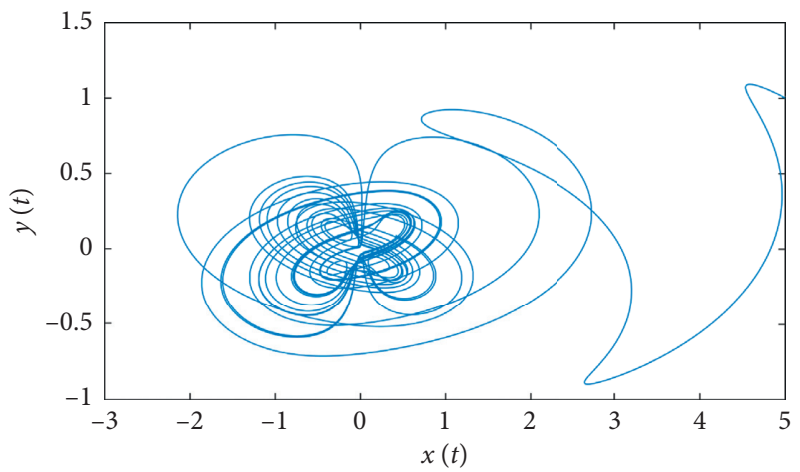

(c)

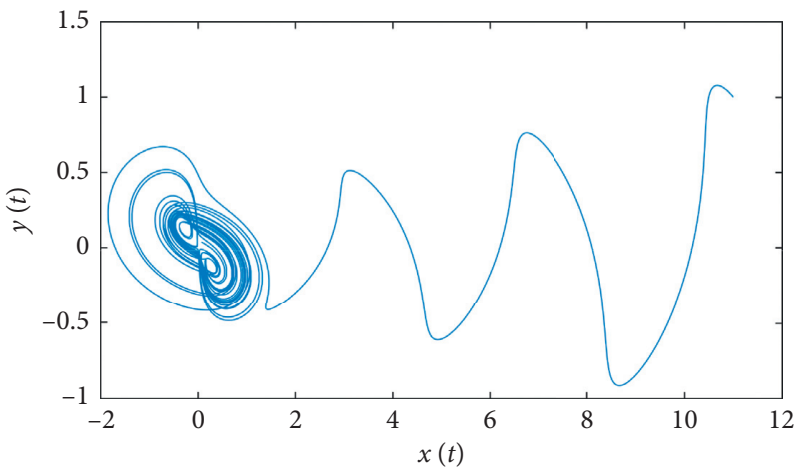

(e)

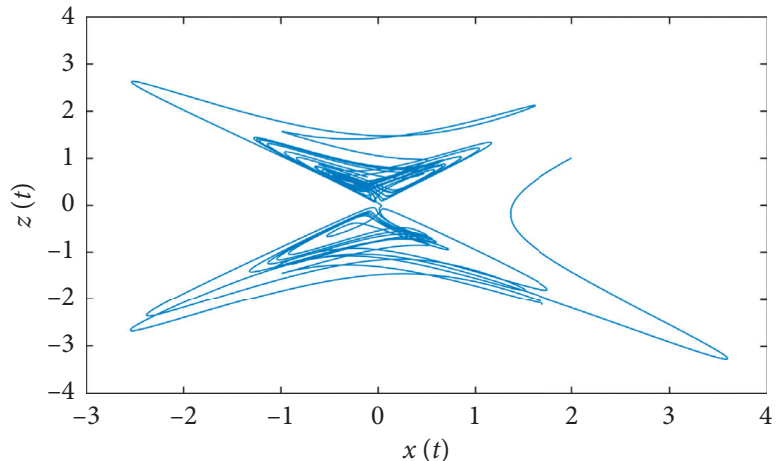

(b)

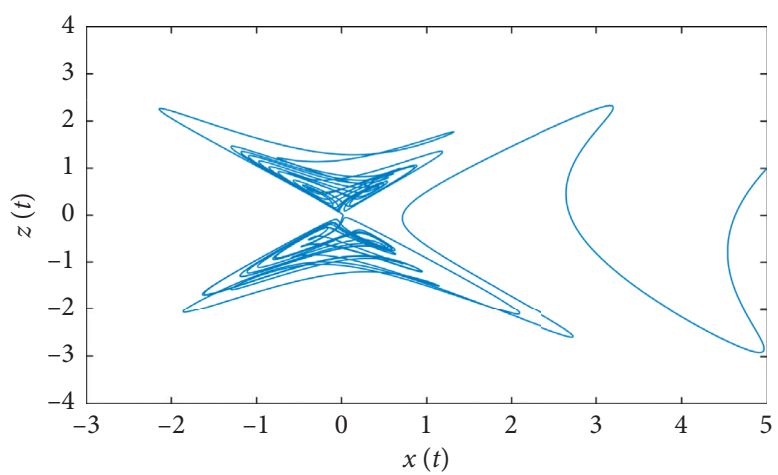

(d)

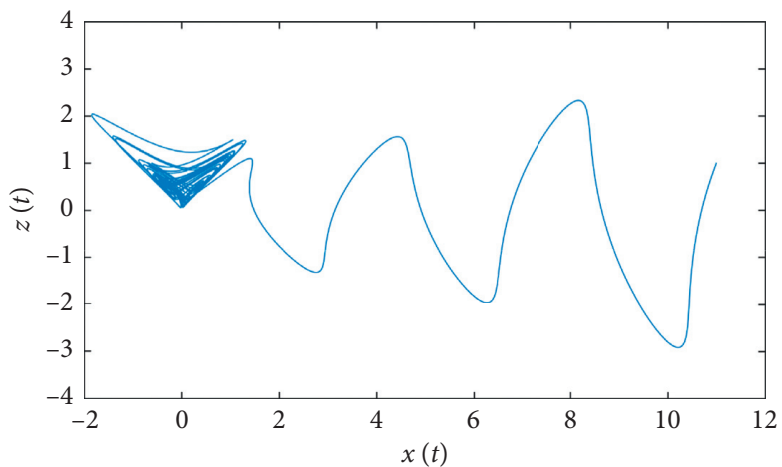

(f)

Figure 1: Chaotic behavior of FOCS (10) with $\alpha=0.95$ in (a) $x-y$ plane with $[x(0), y(0), z(0)]=[2,1,1]$; (b) $x-z$ plane with initial condition $[2,1,1]$; (c) $x-y$ plane with initial condition $[5,1,1]$; (d) $x-z$ plane with initial condition $[5,1,1]$; (e) $x-y$ plane with initial condition $[11,1,1]$; (f) $x-z$ plane with initial condition $[11,1,1]$.

$$
\mathscr{D}^{\alpha} \widehat{\theta}_{i}=\rho_{i} e_{i} \psi_{i}^{T}-\rho_{i} \beta_{i} \widehat{\theta}_{i}
$$

with $\rho_{i}, \beta_{i} \in \mathbb{R}^{+}$.

Based on the above discussion, we can give the following theorem.

Theorem 1. Let the master FOCS be (9) and the slave FOCS be (13) and consider the parametric uncertainties. The synchronization controller is given by (15). The adaptation laws are given by (16). Then, the synchronization error $\varepsilon$ tends to a small neighborhood of the origin determined by design parameters.

Proof. Let

$$
V=\frac{1}{2} \sum_{i=1}^{3} \varepsilon_{i}^{2}+\frac{1}{2} \sum_{i=1}^{4} \frac{1}{\rho_{i}} \widetilde{\theta}_{i}^{T} \widetilde{\theta}_{i} .
$$

According to (17), (18), and Lemma 1, one knows 


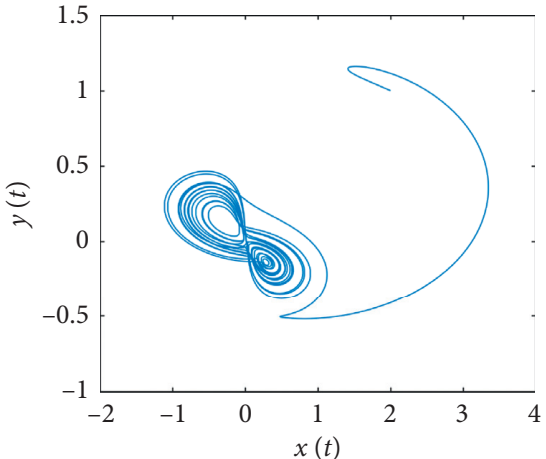

(a)

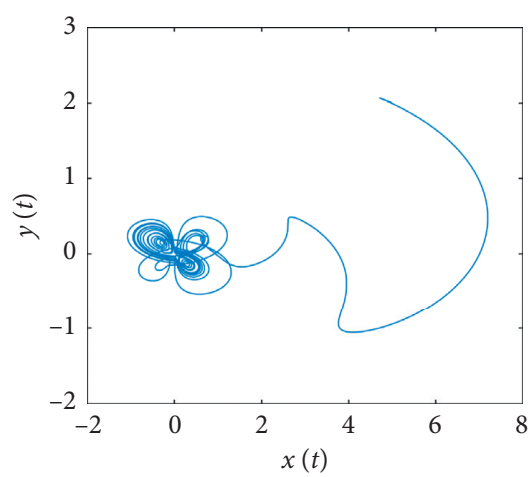

(d)

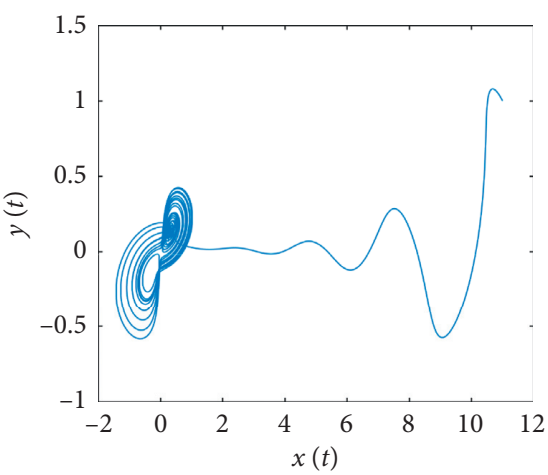

(g)

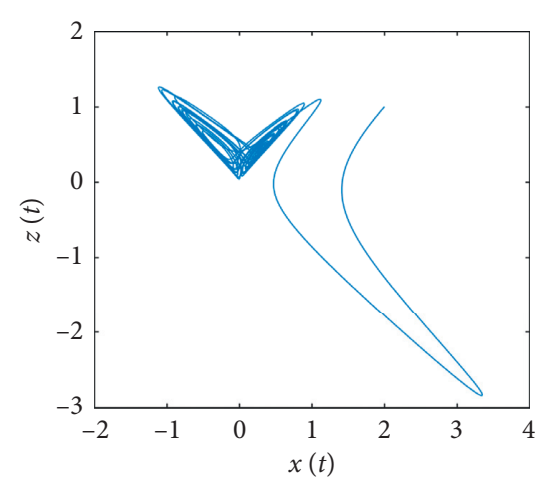

(b)

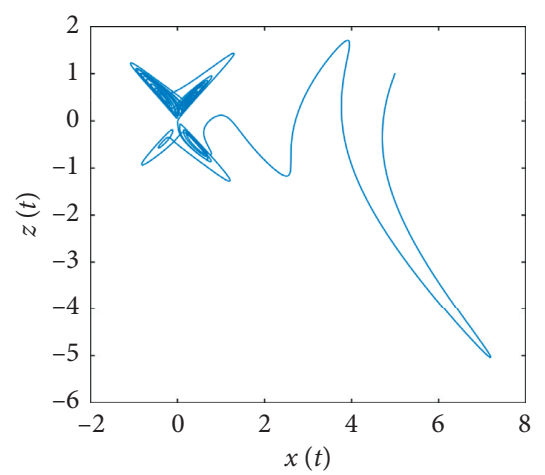

(e)

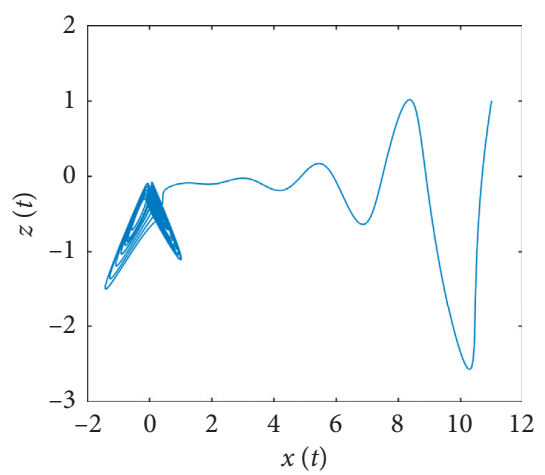

(h)

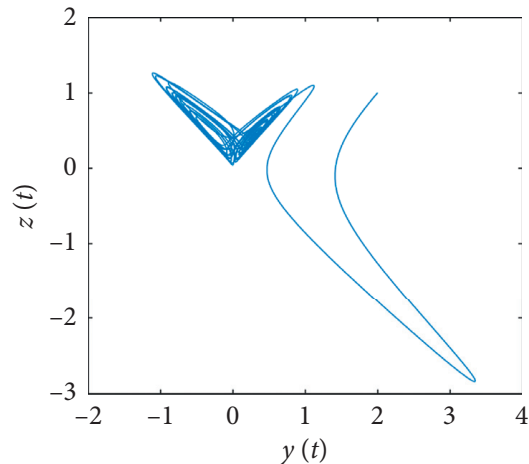

(c)

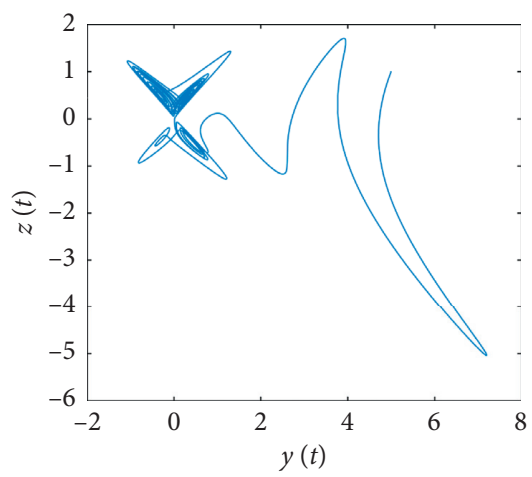

(f)

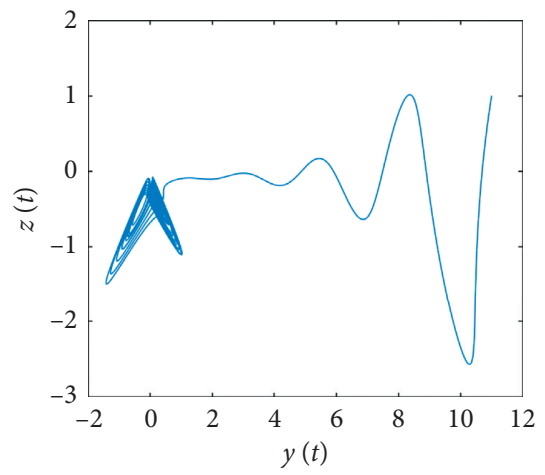

(i)

FIGURE 2: Chaotic behavior of FOCS (10) with $\alpha=0.85$ in (a) $x-y$ plane with initial condition $[2,1,1]$; (b) $x-z$ plane with initial condition $[2,1,1]$; (c) $y-z$ plane with initial condition $[2,1,1]$; (d) $x-y$ plane with initial condition $[5,2,1]$; (e) $x-z$ plane with initial condition $[5,2,1]$; (f) $y-z$ plane with initial condition $[5,2,1]$; (g) $x-y$ plane with initial condition $[11,1,1]$; (h) $x-z$ plane with initial condition $[11,1,1]$; (i) $y-z$ plane with initial condition $[11,1,1]$.

$$
\begin{aligned}
\mathscr{D}^{\alpha} V & =\frac{1}{2} \sum_{i=1}^{3} \mathscr{D}^{\alpha} \varepsilon_{i}^{2}+\frac{1}{2} \sum_{i=1}^{3} \frac{1}{\rho_{i}} \mathscr{D}^{\alpha} \widetilde{\theta}_{i}^{T} \widetilde{\theta}_{i}, \\
& \leq \sum_{i=1}^{3} \varepsilon_{i} \mathscr{D}^{\alpha} \varepsilon_{i}+\sum_{i=1}^{3} \frac{1}{\rho_{i}} \widetilde{\theta}_{i}^{T} \mathscr{D}^{\alpha} \widetilde{\theta}_{i} \\
& =-\sum_{i=1}^{3} \sigma_{i} \varepsilon_{i}^{2}-\sum_{i=1}^{3} \varepsilon_{i} \psi_{i}^{T} \widetilde{\theta}_{i}+\sum_{i=1}^{3} \frac{1}{\rho_{i}} \widetilde{\theta}_{i}^{T} \mathscr{D}^{\alpha} \widetilde{\theta}_{i}+0.2785 \sum_{i=1}^{3} \gamma_{i} .
\end{aligned}
$$



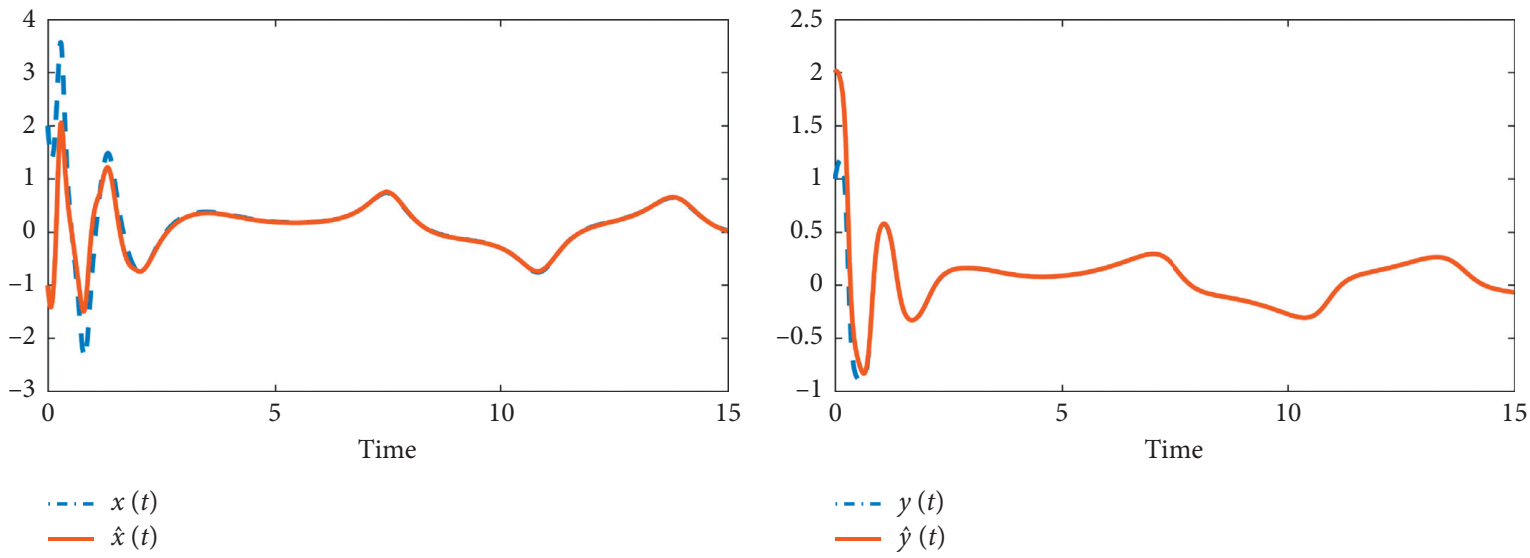

(a)
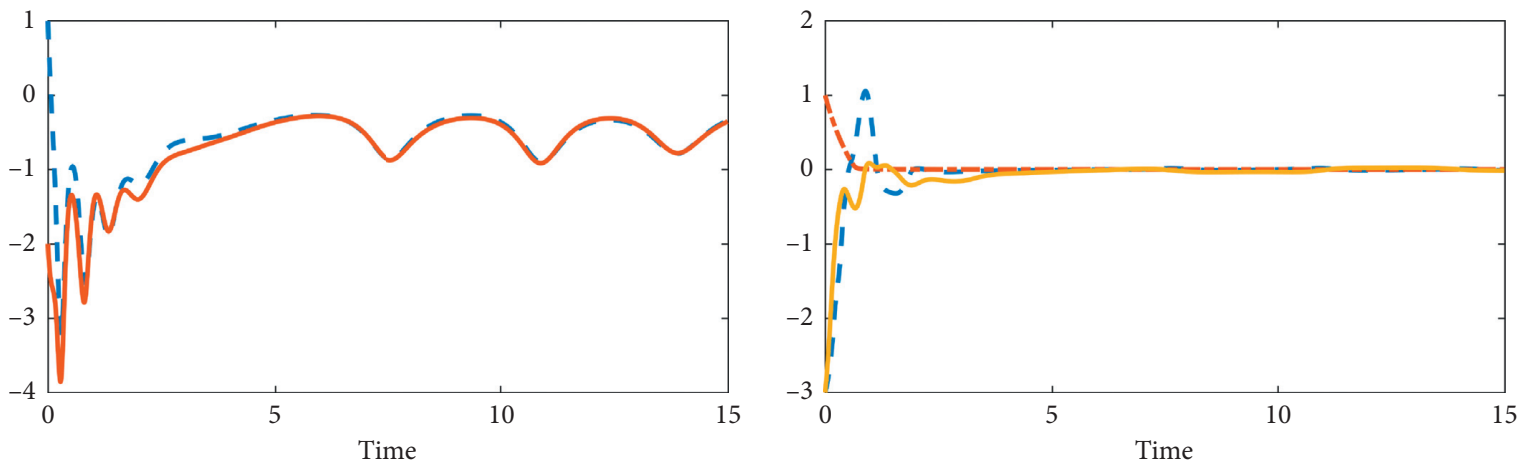

$-\cdot-z(t)$
$-\hat{z}(t)$

$$
\begin{aligned}
--- & \varepsilon_{1}(t) \\
--- & \varepsilon_{2}(t) \\
- & \varepsilon_{3}(t)
\end{aligned}
$$

(c)

(d)

FIgURE 3: Simulation results in (a) $x$ and $\widehat{x}$; (b) $y$ and $\hat{y}$; (c) $z$ and $\widehat{z}$; (d) synchronization errors.

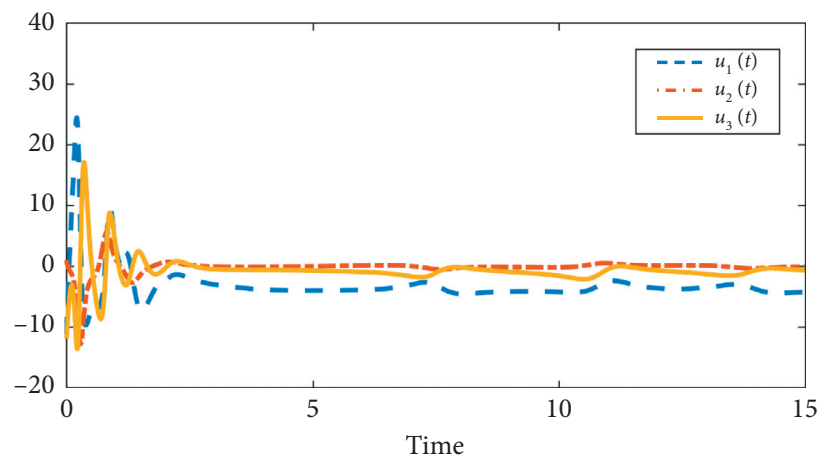

(a)

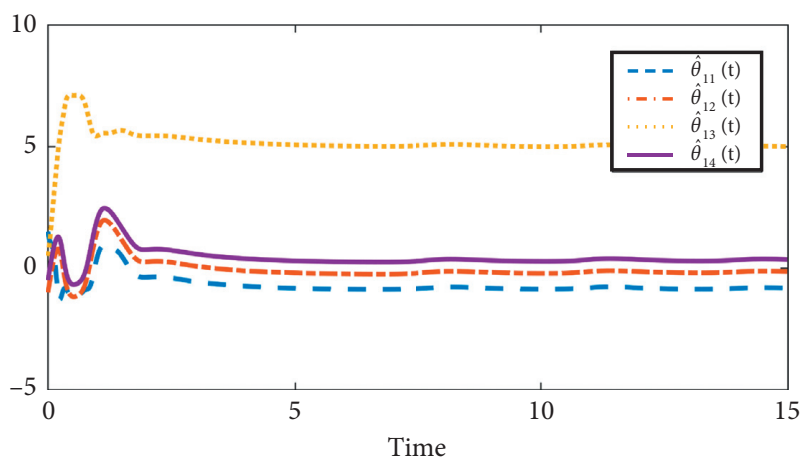

(b)

Figure 4: Continued. 


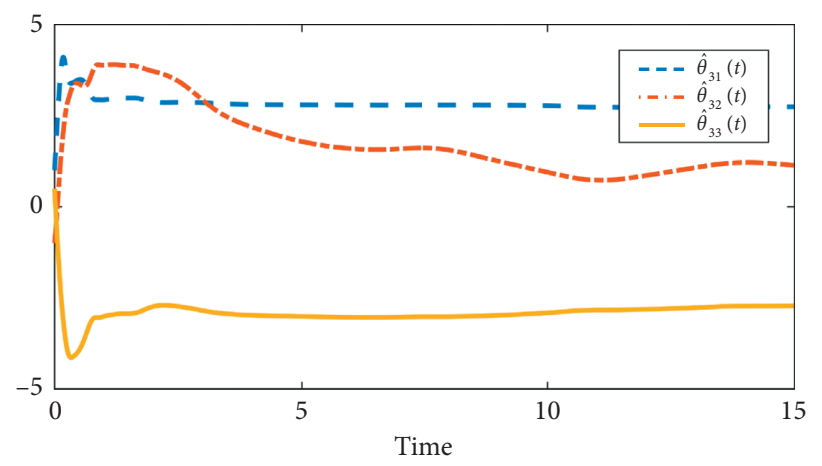

(c)

Figure 4: (a) control inputs; (b) estimation of $\theta_{1}$; (c) estimation of $\theta_{3}$.

Thus, (18) and (20) mean that

$$
\begin{aligned}
\mathscr{D}^{\alpha} V \leq & 0.2785 \sum_{i=1}^{3} \gamma_{i}-\sum_{i=1}^{3} \varepsilon_{i} \psi_{i}^{T} \widetilde{\theta}_{i}+\sum_{i=1}^{3} \tilde{\theta}_{i}^{T}\left[\varepsilon_{i} \psi_{i}^{T}(\zeta)-\beta_{i} \widehat{\theta}_{i}\right] \\
& -\sum_{i=1}^{3} \sigma_{i} \varepsilon_{i}^{2}, \\
= & -\sum_{i=1}^{3} \sigma_{i} \varepsilon_{i}^{2}-\sum_{i=1}^{3} \beta_{i} \widetilde{\theta}_{i}^{T} \widehat{\theta}_{i}+0.2785 \sum_{i=1}^{3} \gamma_{i} \\
= & -\sum_{i=1}^{3} \sigma_{i} \varepsilon_{i}^{2}-\sum_{i=1}^{3} \beta_{i} \tilde{\theta}_{i}^{T}\left(\widetilde{\theta}_{i}+\theta_{i}\right)+0.2785 \sum_{i=1}^{3} \gamma_{i} \\
= & -\sum_{i=1}^{3} \sigma_{i} \varepsilon_{i}^{2}-\sum_{i=1}^{3} \beta_{i} \widetilde{\theta}_{i}^{T} \theta_{i}-\sum_{i=1}^{3} \beta_{i} \tilde{\theta}_{i}^{T} \widetilde{\theta}_{i}+0.2785 \sum_{i=1}^{3} \gamma_{i} \\
\leq & -\sum_{i=1}^{3} \sigma_{i} \varepsilon_{i}^{2}+\sum_{i=1}^{3} \frac{\beta_{i}}{2} \theta_{i}^{T} \theta_{i}-\sum_{i=1}^{3} \frac{\beta_{i}}{2} \widetilde{\theta}_{i}^{T} \widetilde{\theta}_{i}+0.2785 \sum_{i=1}^{3} \gamma_{i} \\
\leq & -\underline{k} \sum_{i=1}^{3} \varepsilon_{i}^{2}-\frac{\beta}{2} \sum_{i=1}^{3} \tilde{\theta}_{i}^{T} \widetilde{\theta}_{i}+\delta_{2} \leq-\delta_{1} V+\delta_{2},
\end{aligned}
$$

with $\underline{\sigma}=\min \left\{\sigma_{1}, \sigma_{2}, \sigma_{3}\right\}, \quad \underline{\beta}=\min \left\{\beta_{1}, \beta_{2}, \beta_{3}\right\}, \quad \bar{\rho}=$ $\max \left\{\rho_{1}, \rho_{2}, \rho_{3}\right\}, \delta_{1}=\min \{2 \underline{k}, \beta \bar{\rho}\}$, and $\delta_{2}=0.2785 \sum_{i=1}^{3} \gamma_{i}+$ $\sum_{i=1}^{3}\left(\beta_{i} / 2\right) \theta_{i}^{T} \theta_{i}$.

Thus, (21) implies

$$
\mathscr{D}^{\alpha} V+h(t)=-\delta_{1} V+\delta_{2},
$$

with $h>0$. Taking Laplace transform on (22) gives

$$
V(s)=\frac{s^{\alpha-1}}{s^{\alpha}+\delta_{2}} V(0)+\frac{s^{-1} \delta_{1}}{\left(s^{\alpha}+\delta_{2}\right)}-\frac{H(s)}{s^{\alpha}+\delta_{2}} .
$$

Then, (23) is solved as

$$
\begin{aligned}
V= & V(0) E_{\alpha, 1}\left(-\delta_{2} t^{\alpha}\right)+\delta_{1} t^{\alpha} E_{\alpha, 1+\alpha}\left(-\delta_{2} t^{\alpha}\right) \\
& -h(t)^{*} t^{-1} E_{\alpha, 0}\left(-\delta_{2} t^{\alpha}\right) .
\end{aligned}
$$

Since $t^{-1} E_{\alpha, 0}\left(-\delta_{2} t^{\alpha}\right) \geq 0$ and $h(t) \geq 0$, one knows that $h^{*} t^{-1} E_{\alpha, 0}\left(-\delta_{2} t^{\alpha}\right) \geq 0$. Then, we obtain

$$
V \leq V(0) E_{\alpha, 1}\left(-B t^{\alpha}\right)+A t^{\alpha} E_{\alpha, 1+\alpha}\left(-B t^{\alpha}\right) .
$$

It is easy to know that

$$
\lim _{t \rightarrow \infty} V(0) E_{\alpha, 1}\left(-\delta_{2} t^{\alpha}\right)=0 .
$$

Then, we know that, for any $\mu \in \mathbb{R}^{+}$, there exists a constant $T_{0} \in \mathbb{R}^{+}$,

$$
V<\mu
$$

holds for all $t>T_{0}$.

As a result, one knows that all signals keep bounded and the synchronization error can be as small as possible for $t>T_{0}$.

Next, let us give the simulation results. Let $\theta_{1}=[1.5,-1.5,2.5,2.0]^{T}, \theta_{3}=[1.5,-1.0,-0.5]^{T}$, and $\theta_{2} \equiv 0$. The related functions are $\phi_{1}=[x, y, z, y+z]^{T}$ and $\varphi_{3}=[x \sin y, \cos z, \sin y]^{T}$. The parameters of the controller and the adaptation law are chosen as $\sigma_{i}=0.5, \rho_{i}=$ 2 , and $\beta_{i}=0.05$ with $i=1,2,3$.

The initial conditions of the master FOCS (10) and the slave FOCS $(13)$ are $[2,1,1]$ and $[-1,2,-2]$, respectively. The fractional order is chosen as $\alpha=0.94$. The simulation results are presented in Figures 3 and 4 . Obviously, as theoretically analyzed, the two FOCSs can be synchronized quickly even in the presence of unmatched parametric uncertainties.

\section{Conclusions}

In this paper, a new three-dimensional FOCS is designed first, and then the dynamic characteristics of this system are analyzed. Theoretical analysis and simulation verification show that the system has complex dynamic behavior and can coexist with different types of multiwing chaotic attractors such as two-wing, three-wing, and four-wing attractors. Based on the fractional-order Lyapunov stability theory, considering uncertain parameters, a robust adaptive synchronous controller is designed. This control method can make the synchronization error arbitrarily small. How to generate a chaotic system with more rings is a research direction in the future. 


\section{Data Availability}

All datasets generated for this study are included in the manuscript.

\section{Conflicts of Interest}

The authors declare that the research was conducted in the absence of any commercial or financial relationships that could be construed as potential conflicts of interest.

\section{Acknowledgments}

This work was supported by the National Natural Science Foundation of China (Grant no. 11302184) and Research Climbing Program of Xiamen Institute of Technology (Grant no. XPDKQ20020).

\section{References}

[1] T. Abdeljawad, "On riemann and caputo fractional differences," Computers \& Mathematics with Applications, vol. 62, no. 3, pp. 1602-1611, 2011.

[2] R. L. Bagley and P. J. Torvik, "A theoretical basis for the application of fractional calculus to viscoelasticity," Journal of Rheology, vol. 27, no. 3, pp. 201-210, 1983.

[3] C. A. Monje, Y. Chen, B. M. Vinagre, D. Xue, and V. FeliuBatlle, Fractional-order Systems and Controls: Fundamentals and Applications, Springer Science \& Business Media, Berlin, Germany, 2010.

[4] A. G. Radwan, K. Moaddy, K. N. Salama, S. Momani, and I. Hashim, "Control and switching synchronization of fractional order chaotic systems using active control technique," Journal of Advanced Research, vol. 5, no. 1, pp. 125-132, 2014.

[5] S. Singh, A. T. Azar, S. Vaidyanathan, A. Ouannas, and M. A. Bhat, Multiswitching Synchronization of Commensurate Fractional Order Hyperchaotic Systems via Active Control," in Mathematical Techniques of Fractional Order Systems, Elsevier, Amsterdam, Netherlands, 2018.

[6] D. Sheng, Y. Wei, S. Cheng, and Y. Wang, "Observer-based adaptive backstepping control for fractional order systems with input saturation," ISA Transactions, vol. 82, pp. 18-29, 2018.

[7] Y. Boukal, M. Darouach, M. Zasadzinski, and N.-E. Radhy, "Robust \$H_\{linfty \}\$ observer-based control of fractionalorder systems with gain parametrization," IEEE Transactions on Automatic Control, vol. 62, no. 11, pp. 5710-5723, 2017.

[8] J. Wang, C. Shao, X. Chen, and Y. Chen, "Fractional-order dob-sliding mode control for a class of noncommensurate fractional-order systems with mismatched disturbances," Mathematical Methods in the Applied Sciences, 2019.

[9] N. Choudhary, J. Sivaramakrishnan, and I. N. Kar, "Sliding mode control of uncertain fractional order systems with delay," International Journal of Control, vol. 93, no. 4, pp. 934-943, 2020.

[10] N. Djeghali, M. Bettayeb, and S. Djennoune, "Sliding mode active disturbance rejection control for uncertain nonlinear fractional-order systems," European Journal of Control, vol. 57, pp. 54-67, 2020.

[11] B. J. Lurie, "Three-parameter tunable tilt-integral-derivative (tid) controller," US Patent US5371670A, 1994.

[12] P. Wang, G. Wen, X. Yu, W. Yu, and Y. Wan, "Synchronization of resilient complex networks under attacks," IEEE
Transactions on Systems, Man, and Cybernetics: Systems, vol. 51, no. 2, pp. 1116-1127, 2019.

[13] Z. Han, S. Li, and H. Liu, "Composite learning sliding mode synchronization of chaotic fractional-order neural networks," Journal of Advanced Research, vol. 25, pp. 87-96, 2020.

[14] Y. Zhou, H. Liu, J. Cao, and S. Li, "Composite learning fuzzy synchronization for incommensurate fractional-order chaotic systems with time-varying delays," International Journal of Adaptive Control and Signal Processing, vol. 33, no. 12, pp. 1739-1758, 2019.

[15] N. Agrawal, A. Kumar, V. Bajaj, and G. K. Singh, "Design of bandpass and bandstop infinite impulse response filters using fractional derivative," IEEE Transactions on Industrial Electronics, vol. 66, no. 2, pp. 1285-1295, 2018.

[16] C. Xu, Y. He, X. Wu, and Y. Mo, "A novel 3d fractional order chaotic system with coexistence of multiple types of multiwing attractors," Journal of Harbin Institutie of Technology, vol. 52, no. 5, pp. 92-98, 2020.

[17] M. Borah and B. K. Roy, "An enhanced multi-wing fractionalorder chaotic system with coexisting attractors and switching hybrid synchronisation with its nonautonomous counterpart," Chaos, Solitons \& Fractals, vol. 102, pp. 372-386, 2017.

[18] X. Yongju, X. Cheng, and X. Changbiao, "New chaotic system and the adaptive synchronization control of its fractional order system," Control Theory \& Applications, vol. 35, no. 6, pp. 878-886, 2018.

[19] Y. Luo, Y. Q. Chen, C. Y. Wang, and Y. G. Pi, “Tuning fractional order proportional integral controllers for fractional order systems," Journal of Process Control, vol. 20, no. 7, pp. 823-831, 2010.

[20] J. Lü, G. Chen, and D. Cheng, "A new chaotic system and beyond: the generalized lorenz-like system," International Journal of Bifurcation and Chaos, vol. 14, no. 05, pp. 15071537, 2004 